\title{
Flares associated with abnormal rotation rates: Longitudinal minimum separation of leading and following sunspots
}

\author{
K. M. Hiremath ${ }^{1}$, G. S. Suryanarayana ${ }^{1}$, and M. R. Lovely ${ }^{1,2, \star}$ \\ ${ }^{1}$ Indian Institute of Astrophysics, Bangalore-560034, India \\ e-mail: hiremath@iiap.res.in \\ 2 Sree Krishna College, Guruvayur, Kerala-680102, India
}

Received 7 December 2004 / Accepted 1 February 2005

\begin{abstract}
Using six years (1969-1974) of data of sunspot groups from the white light pictures of the Kodaikanal Observatory, we compute rotation rates of the leading and the following sunspots and the rate of change of longitudinal separation during their life times. We find that (i) the spots that are associated with abnormal rotation rates (i.e, rotation rates that are greater than $1 \sigma$ from the mean rotation) and that approach at a separation rate of 1-2 deg/day also experience minimum longitudinal separation $\left(\sim 6^{\circ}-10^{\circ}\right)$ of their foot points during the course of their evolution; (ii) spots that have a minimum separation eventually trigger flares; and (iii) events with abnormal rotation rates and minimum approaching distances of the foot points occur on average during between $50-80 \%$ of the life spans, indicating the annihilation of magnetic energy, probably below the surface. These results support the conventional physical scenario of magnetic reconnection that may be responsible for triggering flares.
\end{abstract}

Key words. sunspots - Sun: flares

\section{Introduction}

The conventional picture of the formation of sunspots is that they originate below the solar surface due to an unknown dynamo mechanism. Due to the very high conductivity of the solar plasma, sunspots are glued to the internal plasma and due to buoyancy raise towards the surface. This implies that sunspots are very good tracers of the internal dynamics and structure of the solar interior. Previous studies (Gokhale \& Hiremath 1984; Javaraiah \& Gokhale 1997; Hiremath 2002, and references there in; Sivaraman et al. 2003; Zuccarello \& Zappala 2003) show that variation of the initial rotation rates obtained from the daily motion of sunspot groups with respect to their life spans is similar to the radial variation of the internal rotation profile of the solar plasma.

In order to know whether dynamics of the sunspotsespecially the dynamics due to rotational rates-give clues about the triggering of flares, we computed the daily rotation rates of sunspots (that have leaders and followers) during their life time and we have shown that the abnormal rotation rates of either leading or following spots or both eventually trigger the flares (Hiremath \& Suryanarayana 2003). In that study, because of the strong association between abnormal rotation rates of sunspots and the occurrence of flares, it is possible to estimate the probable region of the depth of magnetic reconnection below the

* On leave under the Faculty Improvement Program. surface. For such reconnection events to occur, a close approach of their foot points and contact of the flux tubes below the surface may be necessary. In the present study, we search for such events and show that triggering of a flare occurs at the time of minimum distance between the leading and the following spots. In Sect. 2, we describe the data used and the method of analysis. Results are presented in Sect. 3. The physical phenomenon of magnetic reconnection that may be responsible for triggering the flare is discussed in Sect. 4 and overall conclusions are presented.

\section{Data and analysis}

For the years 1969-74, we use both the data set of positional measurements (heliographic latitude and longitude from the central meridian) of the sunspot groups (that have leading and following sunspots) taken from daily white light images and the flare events in the $\mathrm{H} \alpha$ images from the Kodaikanal Observatory. The details of the telescope and observations of daily white light images are given by Sivaraman et al. (1992). Using similar criteria (Hiremath 2002) in selecting the sunspot groups, we compute rotation rate $\omega_{i}$ of the leading and following sunspots as follows:

$\omega_{i}=\frac{\left(l_{i+1}-l_{i}\right)}{\left(t_{i+1}-t_{i}\right)}$

where $l$ is the heliographic longitude from the central meridian, $t$ is the time of observation, $i=1,2,3, . . n$, and $n$ is the age of 
Table 1. Kodaikanal observations related to the Greenwich Group numbers.

\begin{tabular}{|c|c|c|c|c|c|}
\hline $\begin{array}{l}\text { Kodai } \\
\text { No. }\end{array}$ & $\begin{array}{l}\text { Green } \\
\text { No. }\end{array}$ & Year & Mon & $\begin{array}{l}\text { Date of } \\
\text { flare }\end{array}$ & $\begin{array}{l}\text { Flare } \\
\text { type }\end{array}$ \\
\hline 13105 & 21482 & 1968 & 1 & 29 & $1-n$ \\
\hline 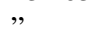 & $"$ & $"$ & 2 & 2 & $s-b$ \\
\hline 13483 & 21894 & 1969 & 2 & 20 & $1-n$ \\
\hline$"$ & $"$ & $"$ & 2 & 25 & $2-b$ \\
\hline$"$ & $"$ & $"$ & 2 & 26 & $2-b$ \\
\hline 13510 & 21936 & 1969 & 3 & 21 & $2-n$ \\
\hline 13621 & 22064 & 1969 & 8 & 2 & \\
\hline$"$ & $"$ & $"$ & 8 & 3 & \\
\hline 13625 & 22068 & 1969 & 8 & 2 & $s-n$ \\
\hline 13640 & 22086 & 1969 & 9 & 26 & $1-b$ \\
\hline 13683 & 22138 & 1969 & 10 & 8 & $1-n$ \\
\hline$"$ & $"$ & $"$ & 10 & 10 & $1-f$ \\
\hline 13696 & 22152 & 1969 & 11 & 2 & $1-f$ \\
\hline 13713 & 22176 & 1969 & 11 & 24 & $2-b$ \\
\hline 13743 & 22210 & 1969 & 12 & 26 & $s-n$ \\
\hline 13776 & $22247 *$ & 1970 & 1 & 17 & $1-n$ \\
\hline 13778 & 22251 & 1970 & 1 & 25 & $1-n$ \\
\hline$"$ & $"$ & $"$ & 1 & 28 & $2-b$ \\
\hline$"$ & $"$ & $"$ & 1 & 30 & $1-n$ \\
\hline 13783 & 22255 & 1970 & 1 & 26 & $1-n$ \\
\hline 13784 & 22261 & 1970 & 1 & 29 & \\
\hline 13791 & 22272 & 1970 & 2 & 9 & $2-b$ \\
\hline$"$ & $"$ & $"$ & 1 & 10 & $1-n$ \\
\hline$"$ & $"$ & $"$ & 1 & 11 & $2-b$ \\
\hline$"$ & $"$ & $"$ & 1 & 12 & $s-b$ \\
\hline 13792 & 22274 & $"$ & 2 & 7 & $1-b$ \\
\hline 13811 & 22291 & 1970 & 2 & 21 & $s-n$ \\
\hline$"$ & $"$ & $"$ & 2 & 25 & $s-n$ \\
\hline 13859 & 22351 & 1970 & 4 & 9 & \\
\hline 13860 & $22349 *$ & 1970 & 4 & 9 & $s-n$ \\
\hline$"$ & $"$ & $"$ & 4 & 11 & $s-n$ \\
\hline$"$ & $"$ & $"$ & 4 & 13 & $s-b$ \\
\hline
\end{tabular}

* Ambiguity in identifying these spot group numbers with Greenwich group numbers.

the spot group. The term rotation rate of the sunspots means the (synodic) angular rotation velocity. We compute daily longitudinal separations $d_{i}=l_{\mathrm{L}}-l_{\mathrm{F}}\left(l_{\mathrm{L}}\right.$ and $l_{\mathrm{F}}$ are the longitudes of the leader and the follower) of the foot points of the spots. Following Eq. (1), we compute the rate of change of longitudinal separation $S_{i}$

$S_{i}=\frac{\left(d_{i+1}-d_{i}\right)}{\left(t_{i+1}-t_{i}\right)}$

In the following analysis we use the combined data (1969-74) for the whole region of heliographic latitudes of $0^{\circ}$ to $40^{\circ}$ in both the solar hemispheres. The combined data set is presented in Tables 1-3. The columns are: (i) Kodaikanal spot group number; (ii) Greenwich spot group number; (iii) the year of observation; (iv) the month of observation; (v) the date of flare occurrence; and (vi) the flare type. From the Kodaikanal data archive, we could not get the flare types for the following Kodaikanal sunspot group numbers: 13621, 13784, 13859 , $13875,14652-14784$. The stars (attached to the Greenwich group numbers) in the second column of Tables 1-3 indicate
Table 2. Kodaikanal observations related to the Greenwich Group numbers.

\begin{tabular}{|c|c|c|c|c|c|}
\hline $\begin{array}{l}\text { Kodai } \\
\text { No. }\end{array}$ & $\begin{array}{l}\text { Green } \\
\text { No. }\end{array}$ & Year & Mon & $\begin{array}{l}\text { Date of } \\
\text { flare }\end{array}$ & $\begin{array}{l}\text { Flare } \\
\text { type }\end{array}$ \\
\hline 13870 & 22362 & 1970 & 4 & 24 & $1-b$ \\
\hline " & $"$ & " & 4 & 25 & $s-n$ \\
\hline 13875 & $22370 *$ & 1970 & 4 & 25 & \\
\hline 13881 & 22379 & 1970 & 5 & 7 & $s-n$ \\
\hline " & $"$ & $"$ & 5 & 8 & $1-n$ \\
\hline 13891 & 22392 & 1970 & 5 & 15 & $s-n$ \\
\hline & $"$ & $"$ & 5 & 16 & $1-b$ \\
\hline 13901 & 22411 & 1970 & 5 & 30 & $1-b$ \\
\hline 13916 & 22433 & 1970 & 6 & 13 & $1-n$ \\
\hline " & $"$ & $"$ & 6 & 14 & $s-n$ \\
\hline ", & $"$ & $"$ & 6 & 16 & $1-n$ \\
\hline 13932 & 22448 & 1970 & 6 & 27 & $s-n$ \\
\hline " & $"$ & $"$ & 6 & 30 & $1-n$ \\
\hline 13937 & 22454 & 1970 & 6 & 30 & $s-n$ \\
\hline " & $"$ & $”$ & 7 & 1 & $s-n$ \\
\hline 13973 & $22495 *$ & 1970 & 8 & 6 & $s-n$ \\
\hline 13980 & 22508 & 1970 & 8 & 24 & $2-n$ \\
\hline 14021 & 22556 & 1970 & 9 & 27 & $1-n$ \\
\hline " & $"$ & $"$ & 9 & 28 & $s-n$ \\
\hline " & $"$ & $"$ & 9 & 29 & $s-n$ \\
\hline 14064 & $22608 *$ & 1970 & 11 & 13 & $1-n$ \\
\hline " & $"$ & $"$ & 11 & 14 & $1-n$ \\
\hline 14108 & 22664 & 1970 & 12 & 1 & $s-n$ \\
\hline 14120 & 22679 & 1971 & 1 & 21 & $1-n$ \\
\hline ", & $"$ & " & 1 & 25 & $1-n$ \\
\hline 14128 & 22686 & 1971 & 1 & 31 & $1-b$ \\
\hline " & $"$ & $"$ & 2 & 3 & $1-n$ \\
\hline 14144 & 22710 & 1971 & 2 & 16 & $1-b$ \\
\hline 14175 & 22738 & 1971 & 3 & 21 & $s-b$ \\
\hline 14184 & 22755 & 1971 & 4 & 11 & $1-n$ \\
\hline
\end{tabular}

${ }^{*}$ Ambiguity in identifying these spot group numbers with Greenwich group numbers.

the ambiguity in identifying the Kodaikanal group numbers with the Greenwich group numbers.

\section{Results}

For the period of observations, we select 57 well-developed spot groups that have leader and follower spots. Using Eqs. (1) and (2), we compute daily rotation rates $\omega_{i}$ and rate of change of longitudinal separation $S_{i}$.

Typical white light images of the evolutionary phase of a spot group that has leading and following sunspots is illustrated in Fig. 1. The spot group grows and decays in the southern hemisphere of the solar disk. Although from the 25th onwards new complex sunspots emerge near the equator, the identity of the leading and the following spots can still be traced unambiguously. In Fig. 2a, we present the rotation rates (in units of deg/day) and the daily longitudinal separation (in units of deg) of the leader and the follower of such a spot group as presented in Fig. 1. In Fig. 2a, the numbers near the vertical lines are the scale values presented along the $y$ axis (rotation and longitudinal separation). In Fig. 2b, we present the rate (in units of degrees/day) of change of longitudinal separation. 
Table 3. Kodaikanal observations related to the Greenwich Group numbers.

\begin{tabular}{|c|c|c|c|c|c|}
\hline $\begin{array}{l}\text { Kodai } \\
\text { No. }\end{array}$ & $\begin{array}{l}\text { Green } \\
\text { No. }\end{array}$ & Year & Mon & $\begin{array}{l}\text { Date of } \\
\text { flare }\end{array}$ & $\begin{array}{l}\text { Flare } \\
\text { type }\end{array}$ \\
\hline 14191 & 22764 & 1971 & 4 & 16 & $2-n$ \\
\hline ", & " & $"$ & 4 & 20 & $2-b$ \\
\hline 14277 & 22877 & 1971 & 8 & 19 & $1-n$ \\
\hline " & $"$ & $"$ & 8 & 27 & $s-n$ \\
\hline 14290 & $22894 *$ & 1971 & 9 & 15 & $1-n$ \\
\hline$"$ & $"$ & $"$ & 9 & 17 & $s-n$ \\
\hline 14322 & 22931 & 1971 & 11 & 16 & $s-n$ \\
\hline$"$ & $"$ & $"$ & 11 & 17 & $s-n$ \\
\hline 14325 & 22940 & 1971 & 12 & 2 & $1-b$ \\
\hline 14381 & 23013 & 1972 & 2 & 15 & $1-b$ \\
\hline$"$ & $"$ & $"$ & 2 & 18 & $s-b$ \\
\hline$"$ & " & $"$ & 2 & 21 & $1-n$ \\
\hline 14384 & 23020 & 1972 & 2 & 18 & $1-n$ \\
\hline$"$ & $"$ & $"$ & 2 & 19 & $s-n$ \\
\hline " & $"$ & $"$ & 2 & 21 & $s-b$ \\
\hline$"$ & $"$ & $"$ & 2 & 24 & $s-n$ \\
\hline$"$ & " & $"$ & 2 & 25 & $s-n$ \\
\hline 14458 & 23110 & 1972 & 5 & 27 & $2-n$ \\
\hline$"$ & $"$ & $"$ & 5 & 28 & $1-n$ \\
\hline$"$ & $"$ & " & 5 & 30 & $1-b$ \\
\hline$"$ & $"$ & $"$ & 6 & 5 & $s-b$ \\
\hline 14462 & 23113 & 1972 & 6 & 5 & $s-n$ \\
\hline 14517 & 23179 & 1972 & 7 & 7 & $2-b$ \\
\hline 14593 & 23272 & 1972 & 11 & 24 & $s-n$ \\
\hline " & ", & $"$ & 11 & 25 & $s-n$ \\
\hline 14635 & 23312 & 1973 & 2 & 5 & $1-n$ \\
\hline 14647 & 23328 & 1973 & 2 & 25 & $s-b$ \\
\hline 14652 & 23332 & 1973 & 3 & $6-7$ & \\
\hline 14657 & 23338 & 1973 & 3 & 20 & \\
\hline$"$ & $"$ & $"$ & 3 & 24 & \\
\hline 14681 & 23377 & 1973 & 6 & $8-10$ & \\
\hline 14712 & 23412 & 1973 & 9 & $2-4$ & \\
\hline 14743 & 23453 & 1973 & 12 & 21 & \\
\hline 14776 & 23491 & 1974 & 4 & 13 & \\
\hline 14784 & 23500 & 1974 & 4 & 26 & \\
\hline
\end{tabular}

${ }^{*}$ Ambiguity in identifying these spot group numbers with Greenwich group numbers.

In Fig. 3, we present the normalized values of daily rotation rates and the rate of change of longitudinal separation. The normalized values are defined as follows. If $x_{i}$ are the data points for different $i$ days, $\bar{x}$ is the average of all data points and $\sigma$ is the standard deviation of rotation rates of the leading and the following spots and the rate of change of separation of their foot points, then the normalized value is $y_{i}=\left(x_{i}-\bar{x}\right) / \sigma$. Since we want to present all three parameters (rotation rates of the leader and the follower and the rate of change of longitudinal separation) that have different ranges of magnitudes, the normalization allows presentation of the three variables in a single plot.

In Fig. 3, whenever there are minimum approaching distances (represented by the negative values of the variation of separation) between the foot points of the leading and the following spots, on the same day or later the spots experience abnormal rotation rates leading to triggering of flares. From the same figure, one can also notice that in order to trigger flares, foot points of the leading and the following sunspots should move towards each other at a rate of 1-2 deg/day.

For the 57 spot groups, we note the occurrence of longitudinal minimum separation and the corresponding occurrence of the flare. The resulting correlative analysis is presented in the scatter diagram of Fig. 4a (left). In 6 years many flares do not satisfy the criterion of a strong association between the minimum longitudinal separation and triggering of the flare. However, we selected only those $\mathrm{H}-\alpha$ flares that correspond to the sunspot groups' heliographic coordinates and time on that day.

Moreover, the correlation coefficient is found to be $94 \%$ with a very high significance $(\sim 100 \%)$. One has to be cautious in interpreting the magnitudes of very high correlation coefficients $(\sim 1)$. In the present analysis we compute the Spearman Rank-Order correlation coefficient and its significance (Press et al. 1992). This method of finding the correlation between two variabilities is more robust than the usual method (i.e., by linear correlation). From this method, we not only find a very high correlation but also at very high significance.

In order to know at what stage of a sunspot's life span the events of minimum separation and flares occur, we separate spot groups of different life spans. In Fig. 4b (right), we present the results with life span along the $x$ axis and the corresponding occurrence of the minimum separation and the flares along the $y$ axis. The errors are determined using the formula $\sigma /(N)^{1 / 2}$, where $N$ is the total number of events of minimum separation and flares and $\sigma$ is the standard deviation. As we found in the previous study (Hiremath \& Suryanarayana 2003), for the events with abnormal rotation rates, a spot with a 4 day life span experiences on average a minimum separation and correspondingly the occurrence of a flare on the second day. A spot with a life span of six days experiences the same events on the third day and so on. In other words, abnormal rotation rates of the spots and the minimum distances of the foot points on average occur at between 50-80\% of the life span during the course of their evolution, probably indicating annihilation of magnetic energy below the surface (Hiremath \& Suryanarayana 2003).

If we assume that the flares occur due to magnetic reconnection, then it is interesting to know the magnitude of minimum separation during the occurrence of the flare. In Fig. 5a, we present the minimum separation (in degrees) of the leading and the following foot points during the occurrence of the flare. In order that reconnection events occur below the surface, the approaching spots that experience abnormal rotation rates should have a minimum longitudinal separation, on average $6^{\circ}-10^{\circ}$ in the photosphere. It is also interesting to know the speed at which foot points of the spots approach each other during the occurrence of the flare. In Fig. 5b, we present the rate of change of minimum separation for different classes of flares. The foot points of the spots that eventually trigger the flares approach each other on average at a rate of $\sim 1^{\circ}-2^{\circ}$ day.

\section{Discussion and conclusions}

Since the majority of spots that have leading and following parts are bipolar (Zirin 1988), we assume that all the spot 

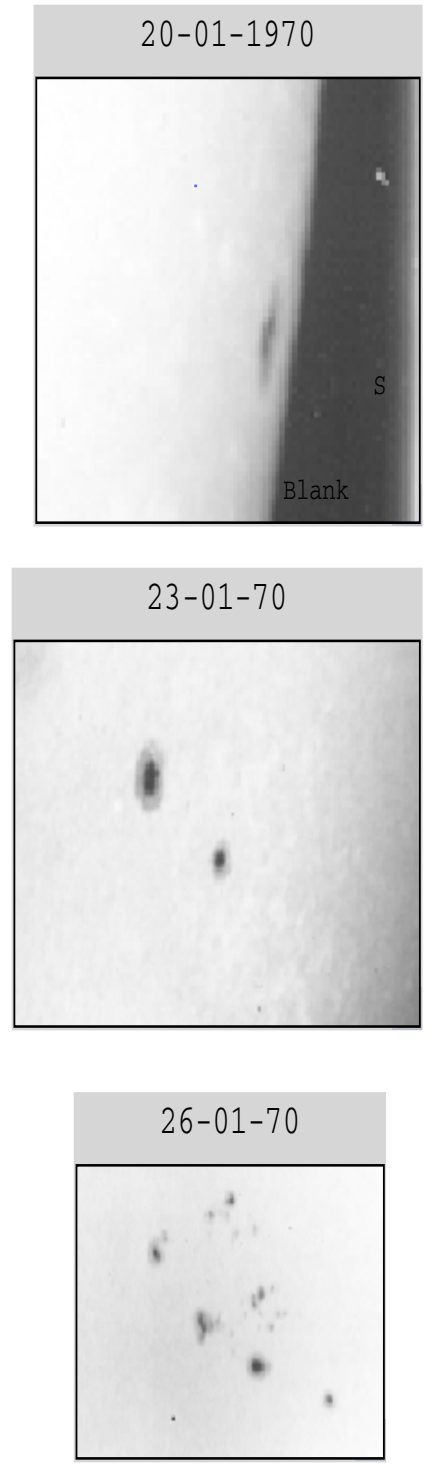
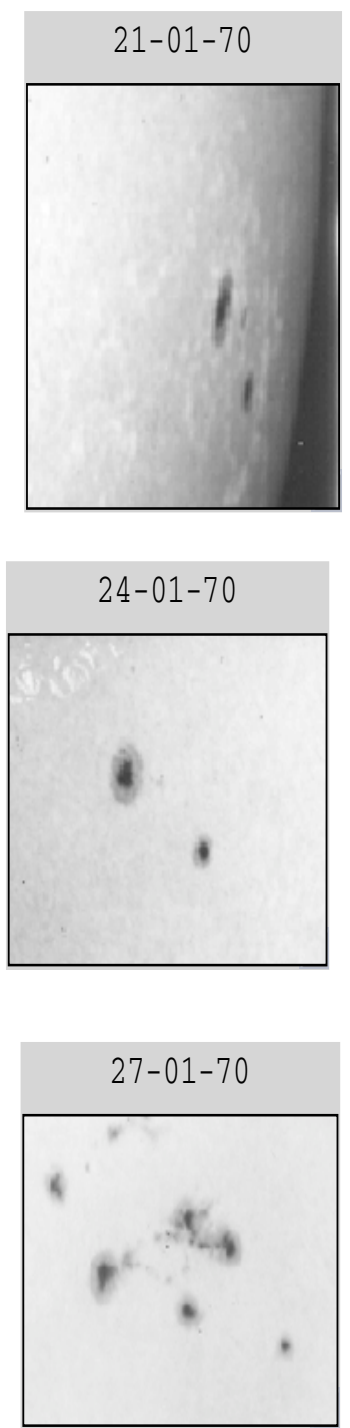
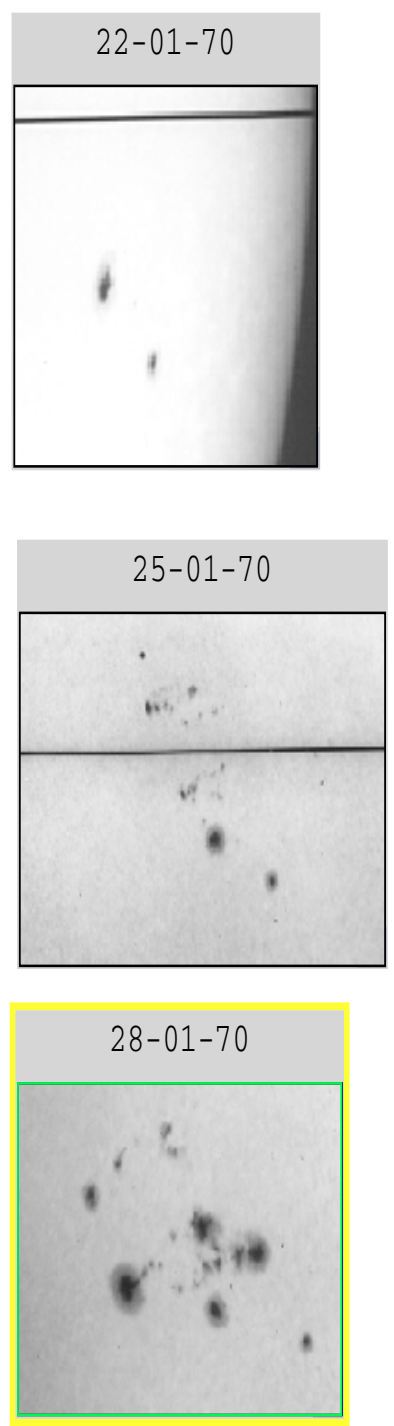

Fig. 1. The evolution of a typical sunspot group that contains leader and follower spots observed from the Kodaikanal Observatory. On the images of the 22 and 25, the horizontal line represents the solar equator. For all the observations, the spot group is south of the equator. The corresponding Greenwich group number for this sunspot group is 22251.

groups that are considered in the present study are bipolar. Thus we can invoke the theory of magnetic reconnection for the interpretation of the results.

Presently it is believed (Priest 1981; Haisch \& Strong 1991; Parker 1994) that the source of energy produced in solar flares is due to magnetic reconnection in a very compact region wherein oppositely directed magnetic fluxes, in the limit of finite electric conductivity, annihilate each other and release the required amount of flare energy. Oppositely directed magnetic flux of large length scale $L$ merges with inflow velocity $v_{\text {in }}$. This merging of flux will form a current sheath. The law of magnetic induction dictates the course of evolution of the plasma. The condition of infinite electric conductivity fails in the region of magnetic field reconnection by producing very high gradients of current and electric fields. Dissipation of these strong currents leads to annihilation of the magnetic field in the region of magnetic reconnection where a steady state exists so that convective and resistive terms in the induction equation are equal. There are two crucial requirements for the reconnecting region that eventually produce the flares. The first requirement is the amount of energy released by the annihilation of the magnetic field $B$ and a cube of length $L$, estimated to be $\sim L^{3} B^{2}$. That means that in order to produce the observed typical flare energy of $\sim 10^{27}-10^{30} \mathrm{erg}$, the length $(L)$ of the reconnecting region below the surface must be $\sim 10^{5}-10^{8} \mathrm{~cm}$ and the strength of the magnetic field should be $10^{5}-10^{3} \mathrm{G}$. The second requirement, from the standard flare mechanism (Petschek 1964), yields the relation $v_{\text {in }}=0.1 v_{\mathrm{a}}$, where $v_{\text {in }}$ is the inflow velocity with which magnetic lines merge and $v_{\mathrm{a}}$ is the Alfven velocity in the vicinity of the magnetic reconnection. From the present study, we satisfy the two requirements as follows. 

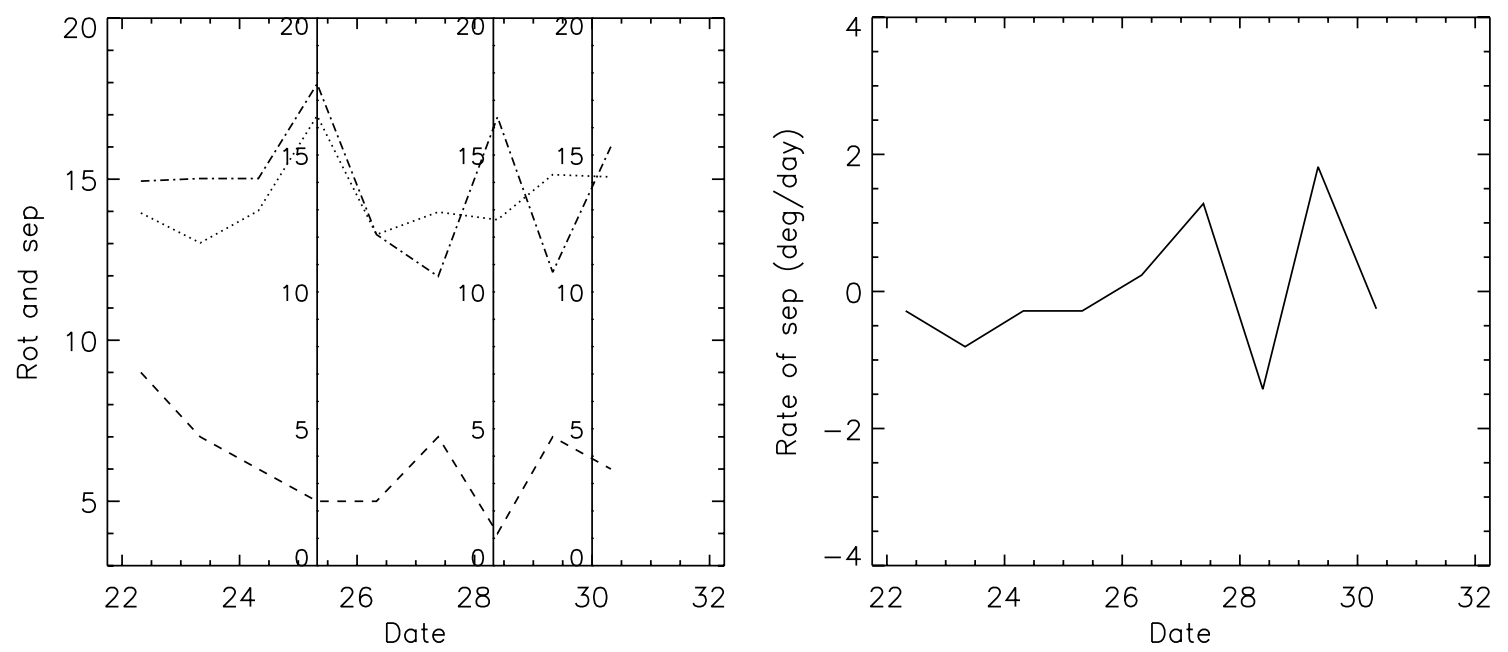

Fig. 2. a) Left: rotation rates and change of longitudinal separation of the leader and the follower during their evolutionary phases. The dotted and dash dotted lines represent the rotation rates (deg/day) of the leading and the following spots. The dashed line represents the change of longitudinal separation (in degrees) of the spots. The vertical continuous lines are the occurrence dates of the flares. The numbers near the vertical lines are the scale values presented on the $y$ axis (rotation and longitudinal separation). b) Right: the typical rate of change of the longitudinal separation (deg/day) of the leader and the follower spots during their evolutionary phases. For both the figures the corresponding Greenwich number of the group is 22251.

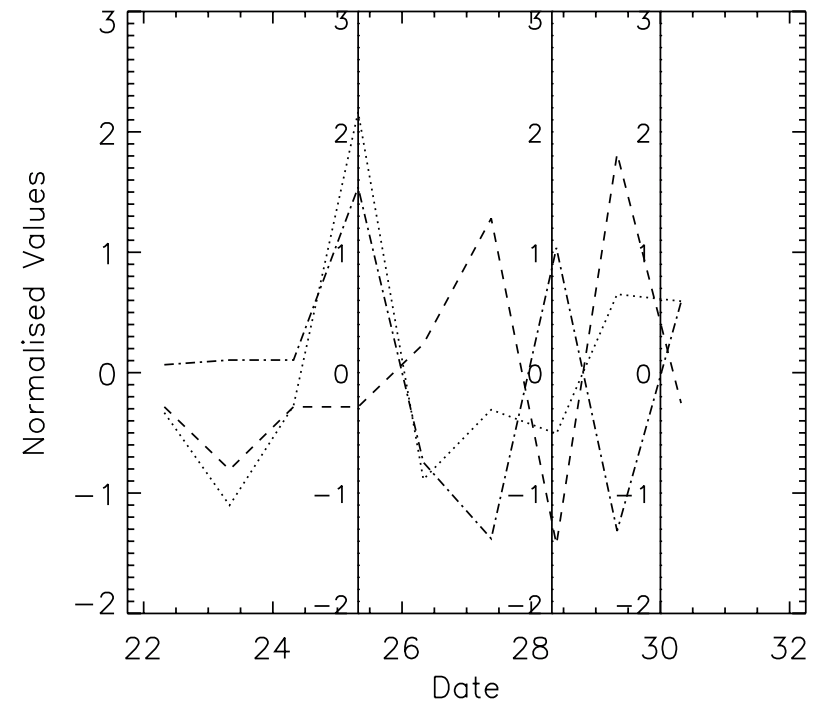

Fig. 3. The normalized rotation rates and rate of change of longitudinal separation of the leader and the follower during their evolutionary phases. The dotted and dash dotted lines represent the normalized rotation rates of the leading and the following spots. The dashed line represents the normalized rate of change of longitudinal separation of the leader and the follower spots. The vertical continuous lines are the occurrence dates of the flares. The corresponding Greenwich number for this sunspot group is 22251 .

To satisfy the first requirement, the present analysis (see the Fig. 5a) shows that on the surface the average minimum separation between bipolar spots is $\sim 6^{\circ}-10^{\circ}$ in longitude $\left(\sim 10^{9} \mathrm{~cm}\right)$ during the occurrence of flare events. By taking a clue from our previous study (Hiremath \& Suryanarayana 2003) that the reconnection may be occurring below the surface at a depth of $0.935 R_{\odot}$, from simple plane trigonometry, one can estimate the thickness (length) of the reconnecting region to be $\sim 10^{5} \mathrm{~cm}$ which is in the required range of $10^{5}-10^{8} \mathrm{~cm}$.
For the second requirement, the strength of the background magnetic field in the vicinity of the reconnecting region is required. The region outside the sunspot has a background magnetic field strength of $\sim 1 \mathrm{G}$ (Stenflo 1994). This is not the same as the strength of the magnetic field $(\sim 40 \mathrm{G})$ of the localized small scale magnetic structures as determined by the Hanle method. On the other hand, we want to determine the strength of the large-scale global magnetic field in the sunspot-free region. Observational (Duvall et al. 1979; Stenflo 1994) and theoretical (Hiremath \& Gokhale 1995) estimates of the magnetic field strength of such a region shows that it is $\sim 1$ Gauss.

Thus, at the surface of the photosphere, in the region outside the sunspot, the Alfven velocity $v_{\mathrm{a}}\left(=B /(4 \pi \rho)^{1 / 2}\right.$, where $B$ is the strength of the magnetic field and $\rho$ is the density) is found to be $\sim 10^{5} \mathrm{~cm} / \mathrm{s}$. The results from Fig. 3 show that the leading and the following spots that approach each other during the occurrence of the flare have a separation velocity of $\sim 1^{\circ} /$ day $\left(10^{4} \mathrm{~cm} / \mathrm{s}\right)$. This result satisfies the requirement that $v_{\text {in }}=0.1 v_{\mathrm{a}}$. Thus, this study strengthens the conventional view that flares may be occurring due to magnetic reconnection.

The overall conclusion of the present study is that during the course of the evolution of leading and following sunspots and in order to trigger flares, the foot points associated with the abnormal rotation rates of the leading and following spots should have an approaching velocity of 1-2 deg/day and ultimately reach a minimum separation of $\sim 6^{\circ}-10^{\circ}$ for probable magnetic reconnection below the surface.

Acknowledgements. We are grateful to the observers of the Kodaikanal Observatory who obtained the white light and $\mathrm{H} \alpha$ images from 1969-1974. We are also grateful to the referee Dr. H. Woehl for the useful comments and suggestions. We thank Mr. P. Michael of the Kodaikanal Observatory for making the prints of the picture (Fig. 1) considered in this study. 

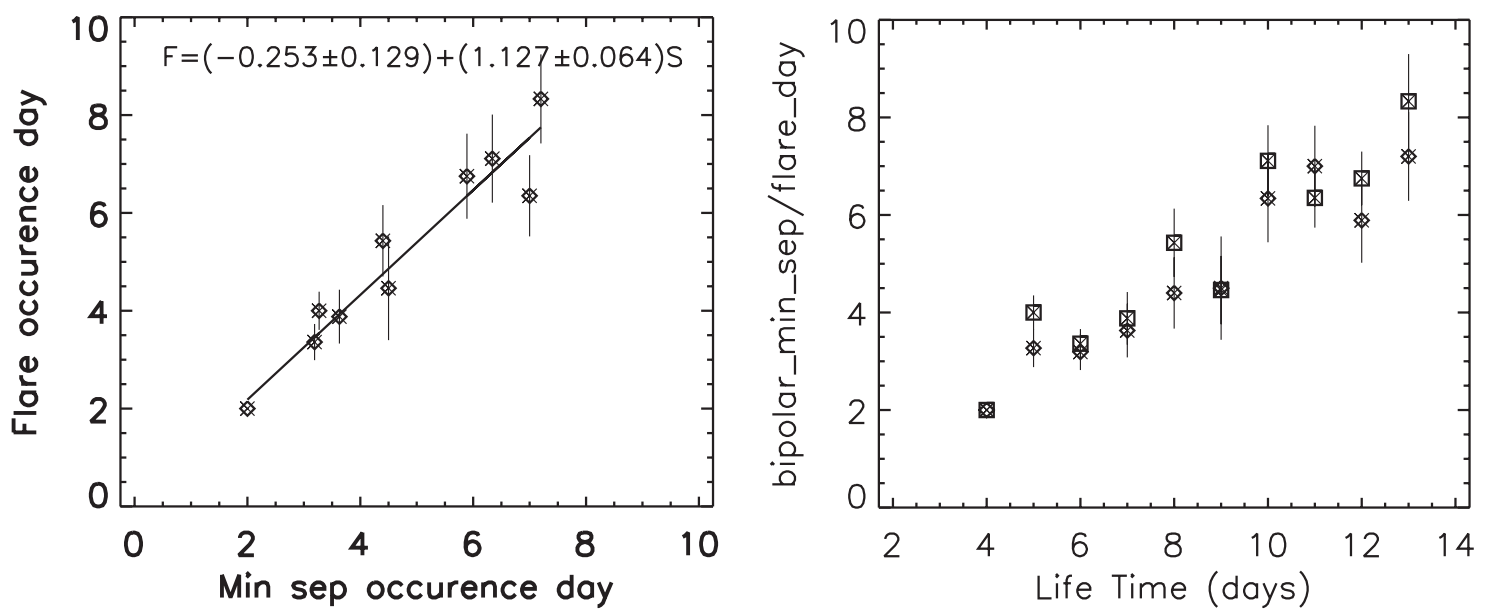

Fig. 4. a) Left: the association between the occurrence of the minimum separation and the flare during the evolution of the spots. The continuous line is obtained from the linear least square fit. Here $S$ and $F$ represent occurrence of minimum separation and the flares respectively. b) Right: days of minimum separation and the flares during the evolution of spots. The symbols $\diamond$ and the square represent the day of minimum separation and the flares respectively.
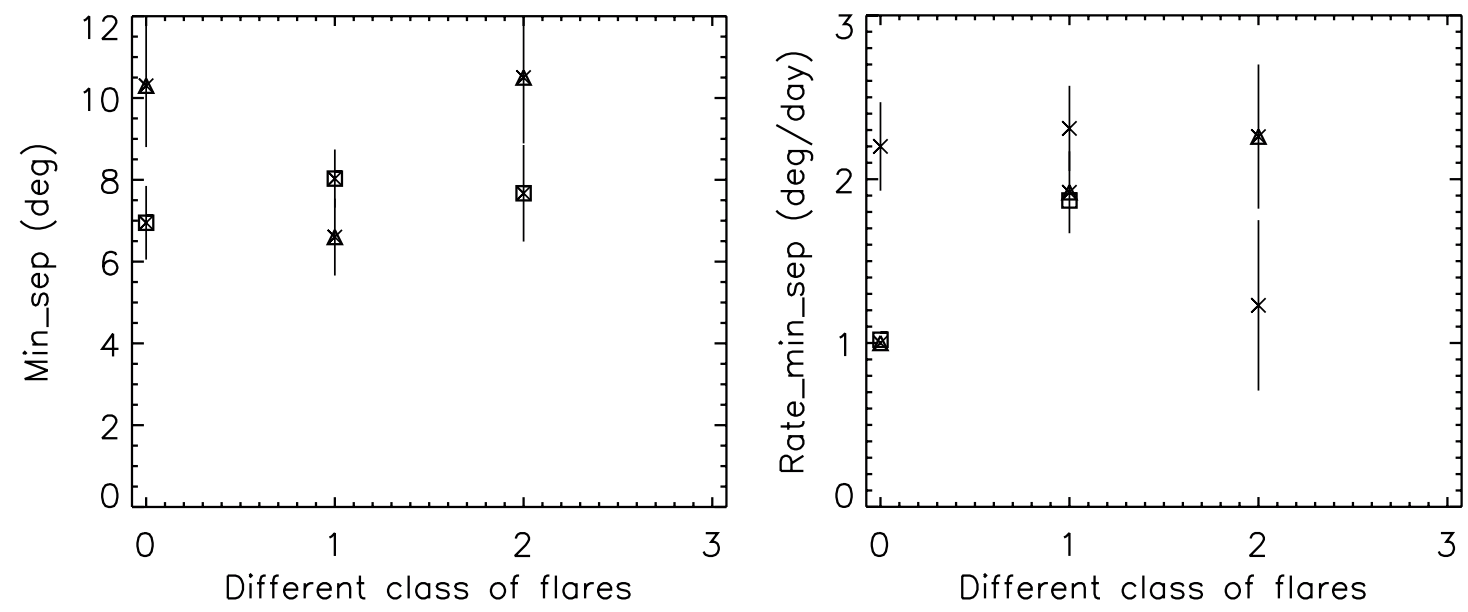

Fig. 5. a) Left: minimum separation for the different classes of flares: the square represents $n$ (normal), the $\Delta$ represents $b$ (bright). Here 0 along the $x$ axis represents the $S$ subclass flare. The numbers 1,2, 3 are higher subclass flares. b) Right: the rate of change of longitudinal minimum separation for different classes of flares: the square represents $f$ (faint), the $\diamond$ is $n$ (normal) and the $\Delta$ represents $b$ (bright). Here 0 along the $x$ axis represents the $S$ subclass flare. The numbers $1,2,3$ are higher subclass flares.

\section{References}

Duvall, T. L., Jr., Scherrer, P. H., Svalgaard, L., \& Wilcox, J. M. 1979, Sol. Phys., 61, 233

Gokhale, M. H., \& Hiremath, K. M. 1984, Bull. Astron. Soc. India., 12,398

Haisch, B., \& Strong, K. T. 1991, Adv. Space Res., 6, 47

Hiremath, K. M., \& Gokhale, M. H. 1995, ApJ, 448, 437

Hiremath, K. M. 2002, A\&A, 386, 674

Hiremath, K. M., \& Suryanarayana, G. S. 2003, A\&A, 411, L497

Javaraiah, J., \& Gokhale, M. H. 1997, A\&A, 137, 63

Parker, E. N. 1994, in Spontaneous Current Sheets in Magnetic fields (Oxford University Press), 286

Petschek, H. E. 1964, AAS-NASA Symp. in the Physics of Solar Flares, ed. W. N. Hess, Washington, DC
Press, W. H., Teukolsky, S. A., Vetterling, W. T., \& Flannery, B. P. 1992, in Numerical Recipes in C (Cambridge University Press), second ed., 640

Priest, E. R. 1981, in Solar Flare Magnetohydrodynamics, ed. E. R. Priest (Gordon and Breach Science Publishers), 14

Sivaraman, K. R., Rausaria, R. R., \& Aleem, S. M. 1992, Sol. Phys., 138,353

Sivaraman, K. R., Hari, S., Gupta, S. S., \& Howard, R. F. 2003, Sol. Phys., 214, 65

Stenflo, J. O. 1994, in Solar Surface Magnetism, ed. R. J. Rutten, \& C. J. Schrijver (Kluwer Academic Publishers), 370

Zirin, H. 1988, in Astrophysics of the Sun (Cambridge University Press), 314

Zuccarello, F., \& Zappala, R. A. 2003, Mem. Della. Soc. Astron. It., 74,619 